\title{
Impedance planimetry values for predicting clinical response following peroral endoscopic myotomy
}

\begin{abstract}
Authors
Robert A. Moran ${ }^{1,2}$, Olaya I. Brewer Gutierrez ${ }^{1}$, Burkhard Rahden ${ }^{3}$, Kenneth Chang ${ }^{4}$, Michael Ujiki ${ }^{5}$, In Kyung Yoo ${ }^{6}$, Shraddha Gulati ${ }^{7}$, John Romanelli ${ }^{8}$, Mohammed Al-Nasser ${ }^{3}$, Toshitaka Shimizu ${ }^{4}$, Mason H. Hedberg ${ }^{9}$, Joo Young Cho ${ }^{6}$, Bu Hayee ${ }^{7}$, David Desilets ${ }^{8}$, Jörg Filser ${ }^{10}$, Kyle Fortinsky ${ }^{4}$, Amyn Haji ${ }^{7}$, Lea Fayad ${ }^{2}$, Omid Sanaei², Mohamad Dbouk², Vivek Kumbhari², Bethany J. Wolf ${ }^{11}$, B. Joseph Elmunzer ${ }^{1}$, Mouen A. Khashab ${ }^{2}$
\end{abstract}

Institutions

1 Division of Gastroenterology and Hepatology, Medical University of South Carolina, Charleston, South Carolina, United States

2 Division of Gastroenterology and Hepatology, Johns Hopkins Hospital, Baltimore, Maryland, United States

3 Department of Surgery Paracelsus, Medical Private Unviersity, Salzburg, Austria

4 Department of Gastroenterology, University of California Irvine Health, Irvine, California, United States

5 Department of Gastroenterology, North Shore University Health System, Evanston, Illinois, United States

6 Digestive Disease Center, CHA Bundang Medical Center, CHA University, Seongnam, Republic of Korea

7 Department of Gastroenterology, King's College Hospital, London, United Kingdom

8 Department of Gastroenterology, University of Massachusetts Medical School/Baystate, Baystate Medical Center, Springfield, Massachusetts, United States

9 Department of Gastroenterology, University of Chicago Medical Center, Chicago, Illinois, United States

10 Department of Surgery, InnKlinikum Altötting, Altötting, Germany

11 Department of Public Health Sciences, Medical University of South Carolina, Charleston, South Carolina, United States

submitted 8.1.2020

accepted after revision 28.7.2020

published online 4.11 .2020

Bibliography

Endoscopy 2021; 53: 570-577

DOI 10.1055/a-1268-7713

ISSN 0013-726X

(c) 2020. Thieme. All rights reserved.

Georg Thieme Verlag KG, Rüdigerstraße 14, 70469 Stuttgart, Germany

Figs. $1-3$

Supplementary material is available under https://doi.org/10.1055/a-1268-7713
Corresponding author

Mouen A. Khashab, MD, Division of Gastroenterology and Hepatology, Johns Hopkins Hospital, 1800 Orleans Street, Suite 7125G, Baltimore, MD 21287, United States

Fax: +1-443-683-8335

mkhasha1@jhmi.edu

\section{ABSTRACT}

Background There is growing interest in developing impedance planimetry as a tool to enhance the clinical outcomes for endoscopic and surgical management of achalasia. The primary aim of this study was to determine whether impedance planimetry measurements can predict clinical response and reflux following peroral endoscopic myotomy (POEM).

Methods A multicenter cohort study of patients with achalasia undergoing POEM was established from prospective databases and retrospective chart reviews. Patients who underwent impedance planimetry before and after POEM were included. Clinical response was defined as an Eckardt score of $\leq 3$. Tenfold cross-validated area under curve (AUC) values were established for the different impedance planimetry measurements associated with clinical response and reflux development.

Results Of the 290 patients included, 91.7\% (266/290) had a clinical response and 39.4\% (108/274) developed reflux following POEM. The most predictive impedance planimetry measurements for a clinical response were: percent change in cross-sectional area (\% $\triangle \mathrm{CSA}$ ) and percent change in distensibility index $(\% \Delta \mathrm{DI})$, with AUCs of 0.75 and 0.73 , respectively. Optimal cutoff values for $\% \Delta C S A$ and $\% \Delta D I$ to determine a clinical response were a change of $360 \%$ and $272 \%$, respectively. Impedance planimetry values were much poorer at predicting post-POEM reflux, with AUCs ranging from 0.40 to 0.62 .

Conclusion Percent change in CSA and distensibility index were the most predictive measures of a clinical response, with a moderate predictive ability. Impedance planimetry values for predicting reflux following POEM showed weak predictive capacity. 


\section{Introduction}

There is growing interest in developing impedance planimetry, an endoscopic device that measures esophagogastric junction (EGJ) distensibility, as a tool to optimize the technical aspects and clinical outcomes of endoscopic and surgical treatments for achalasia [1].

Disruption of the hypertensive lower esophageal sphincter is the primary target for therapies directed at treating achalasia. Impedance planimetry directly measures pressure and crosssectional surface area (CSA) at the sphincter. The distensibility index is an indirect measurement, calculated by dividing the CSA by pressure. A low distensibility index accurately predicts the presence of achalasia and has been widely incorporated into the diagnostic toolbox for this disease [2]. Based on preliminary data in patients undergoing peroral endoscopic myotomy (POEM) for the management of achalasia, higher post-procedural CSA and distensibility index measurements are associated with a favorable clinical response [3-6]. Importantly, these higher values are also associated with higher rates of reflux, an important long-term consequence of disrupting the lower esophageal sphincter $[3,5]$. In the published literature, there is a large variation in the reported "optimal range" of impedance planimetry measurements that are most predictive of a clinical response [5-7]. This is a likely consequence of the low numbers of patients included in previous studies, which are mainly single-operator and/or single-center studies. To improve clinical outcomes of patients undergoing treatment for achalasia, this "optimal range" needs to be clearly defined.

The primary aim of this study was to evaluate which impedance planimetry measurements are most predictive of clinical outcomes in patients undergoing POEM for the treatment of achalasia.

\section{Methods}

This was a retrospective, international, multicenter cohort study including 11 tertiary centers (two from the USA, seven from Europe, two from Asia). Institutional review board approval was obtained at all institutions. Patients were enrolled from April 2013 to December 2017. Data were obtained from a combination of prospective databases and retrospective chart reviews. The current study included 43 patients from the Johns Hopkins Hospital and University Hospital Wurzburg datasets who had also been included in a previously published study exploring the use of impedance planimetry in predicting outcomes following POEM [3].

\section{Inclusion/exclusion criteria}

For inclusion in the study, patients had to meet all of the following inclusion criteria: 1 ) age $\geq 18$ years at the time of POEM; 2 ) achalasia subtype I or II based on the Chicago classification [8]; 3) performance of impedance planimetry at a balloon volume of $30 \mathrm{~mL}$ both before and after POEM; and 4) a minimum of 3 months' follow-up. Patients were excluded if they underwent POEM for treatment of achalasia type III or EGJ outlet obstruction or any other esophageal motility disorder.

\section{POEM technique}

The POEM technique has been described previously in multiple studies [9]. There were variations in the technique and the post-procedure protocol employed among different centers. Prior to the commencement of the study, participating centers had completed between 50 and 200 POEM procedures. All patients underwent POEM under general anesthesia, with paralysis initiated at the discretion of the endoscopist. Patients were admitted overnight after POEM and kept nil by mouth until an esophagram with water-soluble contrast was performed the day after the procedure to rule out extra-esophageal leakage of contrast. This protocol was observed in all but one institution (CHA Bundang Medical Center), where patients underwent an esophagram immediately following the procedure and were discharged home the same day. Patients were followed up in an outpatient clinic or by a phone call to assess symptomatic response to POEM.

\section{Technique for impedance planimetry measurements}

Impedance planimetry measurements were acquired using an endoluminal functional lumen imaging probe (endoFLIP; Medtronic GI Solutions, Sunnyvale, California, USA). This technique has been described previously [3]. Preprocedure (intraprocedurally, prior to the mucosotomy) and post-procedure (immediately following the myotomy and prior to the mucosotomy closure), the FLIP catheter was advanced to the EGJ and inflated to $30 \mathrm{~mL}$. The catheter was centered at the EGJ and then left in place for 30-60 seconds prior to recording the impedance planimetry measurements. The EGJ distensibility index was defined as the smallest CSA divided by the pressure at the corresponding point.

\section{Impedance planimetry measurements}

The following impedance planimetry measurements were obtained from the lower esophageal sphincter: 1) CSA in mm; 2) balloon pressure in $\mathrm{mmHg}$; 3) distensibility index in $\mathrm{mm} /$ $\mathrm{mmHg}$; 4) change in CSA ( $\triangle \mathrm{CSA}$ defined as post-procedural CSA minus preprocedural CSA); 5 ) change in pressure ( $\triangle P r$ defined as post-procedural pressure minus preprocedural pressure); 6) change in distensibility index ( $\Delta \mathrm{DI}$ defined as post-procedural distensibility index minus preprocedural distensibility index); 7) percent change in CSA (\% $\triangle$ CSA defined as post-procedural CSA divided by preprocedural (SA); 8) percent change in pressure (\% $\triangle P r$ defined as post-procedural pressure divided by preprocedural pressure); 9) percent change in distensibility index (\% $\Delta \mathrm{DI}$ defined as post-procedural distensibility index divided by preprocedural distensibility index).

\section{Definition of clinical variables}

A clinical response was defined as an Eckardt score of $\leq 3$ on last follow-up after POEM [10]. Patients were diagnosed with gastroesophageal reflux disease (GERD) symptoms based on the presence of typical symptoms. Esophagitis was defined as the presence of a Los Angeles grade of at least grade A esophagitis 
on upper endoscopy [11]. An abnormal pH/impedance test was defined as a Demeester score of $>14.72$.

\section{Statistical analysis}

Descriptive statistics were calculated for all patient characteristics. The primary goal was to evaluate univariate prediction performance of nine impedance planimetry parameters for predicting patient outcomes (i.e. clinical response, occurrence of abnormal $\mathrm{pH} /$ impedance testing and or esophagitis on upper endoscopy, and development of post-POEM GERD symptoms). Univariate models between each outcome with the nine impedance planimetry physiological parameters were developed using a generalized estimating equation models (GEEs). All GEE models assumed a binary distribution with a logit link function and included a fixed effect for the nine impedance planimetry physiological parameters being considered and a random center effect to account for clustering within center. Model assumptions for each outcome and planimetry parameter were assessed and transformations were considered as needed. Additionally, all GEE models were fit using stratified 10-fold cross validation to ensure similar outcome distributions within fold. Ten-fold cross validation splits the data into 10 equally sized data sets and then builds a model on 9/10th of the data and predicts on the $1 / 10$ th excluded during model development. This process is repeated 10 times for all 10 folds of the data. Prediction performance of each planimetry parameter was assessed using the 10 -fold cross-validated area under the receiver operating characteristics (ROC) curve (cvAUC), calculated using the predicted probabilities from the validation data excluded in fitting the 10 -fold models. The $95 \%$ confidence intervals (Cls) for the cvAUC were calculated using the method proposed by LeDell et al. [12]. As an exploratory analysis, we also considered multivariate prediction models considering all nine planimetry parameters and used the GEEBoost algorithm to conduct simultaneous model fitting and variable selection $[13,14]$. Prediction performance was assessed as described for the univariate models. Complete planimetry and response data were available for all study patients.

Secondary measures of prediction performance, including sensitivity, specificity, positive predictive value (PPV), and negative predictive value (NPV), were determined for multiple cutoff values of those parameters with the largest AUC for each outcome to determine optimal test characteristics for clinical response and GERD. The optimal cut-point of impedance planimetry values used to predict clinical response were calculated by identifying the cut-point that yielded the maximum Youden index. Owing to the limited number of patients across centers who did not exhibit the primary clinical response, cross-validation was not feasible for evaluating potential cut-points for the different impedance planimetry variables; therefore all data were used in estimating test characteristics at different cutpoints.

All analyses were conducted in SAS v. 9.4 (SAS Institute, Cary, North Carolina, USA) and R v. 3.5.2 (The Comprehensive $\mathrm{R}$ Archive Network; R Foundation for Statistical Computing, Vienna, Austria. https://www.r-project.org/).

\section{Results}

The study population included 290 patients. There were more female patients $(53.1 \%$ ) and the mean age was 47.2 years (standard deviation 17.4 years). Overall, $35.5 \%$ of patients had received some prior treatment, with the most common being pneumatic dilation ( $26.0 \%$ of patients). The median pre-POEM Eckardt score was 8 and the median post-POEM Eckardt score was 1. Patient and study characteristics are shown in $>$ Table 1.

\section{Prediction of clinical response}

The majority ( $n=266,91.7 \%$ ) of patients achieved a clinical response (Eckardt score of $\leq 3$ ) following POEM. The median time from POEM to calculation of the Eckardt score was 36.3 weeks (interquartile range 36.2 ). The cvAUC values for the different univariate models ranged from 0.55 to 0.75 ( $\triangleright$ Table 2$)$. The clinical parameters of $\Delta C S A, \% \Delta C S A$, and $\% \Delta D I$ all had estimated $A U C s>0.7$, suggesting moderate prediction performance. The ROC curves for the complete data and based on the average obtained from the 10 -fold cross validation (cvAUC) for

- Table 1 Patient and study characteristics $(n=290)$.

\begin{tabular}{|c|c|}
\hline \multicolumn{2}{|l|}{ Characteristic } \\
\hline Male sex, n (\%) & $136(46.9)$ \\
\hline Age, mean (SD), years & $47.2(17.4)$ \\
\hline \multicolumn{2}{|l|}{ Achalasia type, n (\%) } \\
\hline - Type I & $69(23.8)$ \\
\hline - Type II & $221(76.2)$ \\
\hline Pretreatment IRP, mean (SD), mmHg & $28.5(15.7)$ \\
\hline Prior treatment, n (\%) & $103(35.5)$ \\
\hline - Botox injections & $33(11.4)$ \\
\hline - Pneumonia dilations & $75(25.9)$ \\
\hline - Heller myotomy & $19(6.6)$ \\
\hline - POEM & $5(1.7)$ \\
\hline \multicolumn{2}{|l|}{ Eckardt scores } \\
\hline - Pre-POEM, median (IQR) & $8(3)$ \\
\hline - Post-POEM, median (IQR) & $1(1)$ \\
\hline \multicolumn{2}{|l|}{ Symptom duration, n (\%) } \\
\hline - <1 year & $71(24.5)$ \\
\hline - $>1$ year to $<2$ years & $63(21.7)$ \\
\hline - $>2$ year and $<3$ years & $38(13.1)$ \\
\hline - $>3$ years and $<5$ years & $33(11.4)$ \\
\hline - $>5$ years and $<10$ years & $40(13.8)$ \\
\hline - >10 years & $45(15.5)$ \\
\hline Myotomy, anterior orientation, n (\%) & $228(78.6)$ \\
\hline $\begin{array}{l}\text { SD, standard deviation; IRP, integrated re } \\
\text { endoscopic myotomy; IQR, interquartile }\end{array}$ & Ire; POEM, peroral \\
\hline
\end{tabular}


- Table 2 Tenfold cross-validated area under the receiver operating characteristics curves for generalized estimating equation models for the three clinical outcomes by each of the nine impedance planimetry measures.

\begin{tabular}{|c|c|}
\hline Outcomes and predictor & cvAUC ( $95 \% \mathrm{Cl})$ \\
\hline \multicolumn{2}{|l|}{ Clinical response (Eckardt score $\leq 3$ ) } \\
\hline - Post-procedure pressure & $0.55(0.44-0.67)$ \\
\hline " $\Delta \mathrm{Pr}$ & $0.66(0.57-0.75)$ \\
\hline . $\% \Delta \operatorname{Pr}$ & $0.65(0.56-0.74)$ \\
\hline - Post-procedure CSA & $0.58(0.49-0.68)$ \\
\hline - $\triangle C S A$ & $0.72(0.62-0.81)$ \\
\hline - $\% \Delta C S A$ & $0.75(0.67-0.89)$ \\
\hline - Post-procedure distensibility index & $0.55(0.47-0.64)$ \\
\hline$\cdot \Delta \mathrm{DI}$ & $0.68(0.58-0.77)$ \\
\hline . $\% \Delta \mathrm{DI}$ & $0.73(0.63-0.82)$ \\
\hline \multicolumn{2}{|c|}{ Abnormal pH impedance and/or esophagitis } \\
\hline - Post-procedure pressure & $0.53(0.45-0.61)$ \\
\hline - $\Delta \mathrm{Pr}$ & $0.40(0.32-0.47)$ \\
\hline . $\% \Delta P r$ & $0.51(0.43-0.58)$ \\
\hline - Post-procedure CSA & $0.59(0.52-0.67)$ \\
\hline - $\triangle C S A$ & $0.61(0.54-0.69)$ \\
\hline - $\% \triangle C S A$ & $0.62(0.55-0.70)$ \\
\hline - Post-procedure distensibility index & $0.60(0.53-0.68)$ \\
\hline . $\Delta \mathrm{DI}$ & $0.59(0.51-0.67)$ \\
\hline . \% $\% \mathrm{DI}$ & $0.58(0.50-0.66)$ \\
\hline \multicolumn{2}{|l|}{ Post-procedure GERD } \\
\hline - Post-procedure pressure & $0.55(0.48-0.62)$ \\
\hline - $\Delta \mathrm{Pr}$ & $0.51(0.44-0.57)$ \\
\hline . \% $\Delta P r$ & $0.53(0.45-0.60)$ \\
\hline - Post-procedure CSA & $0.62(0.56-0.68)$ \\
\hline - $\triangle \mathrm{CSA}$ & $0.59(0.52-0.65)$ \\
\hline - \% $\triangle \mathrm{CSA}$ & $0.43(0.37-0.50)$ \\
\hline - Post-procedure distensibility index & $0.58(0.51-0.64)$ \\
\hline . $\Delta \mathrm{DI}$ & $0.57(0.50-0.63)$ \\
\hline . \% $\% \mathrm{DI}$ & $0.49(0.45-0.55)$ \\
\hline
\end{tabular}

cVAUC, 10 fold cross-validated area under the receiver operating characteristics curve; $\mathrm{Cl}$, confidence interval; $\mathrm{Pr}$, pressure; $\Delta$, change in parameter; $\% \Delta$, percent change in parameter; CSA, cross-sectional area; $\mathrm{DI}$, distensibility index; GERD; gastroesophageal reflux disease.

these three parameters are presented in $>$ Fig. $\mathbf{1}$, and $>$ Table 3 provides sensitivity, specificity, PPV, and NPV for different cutoffs for these three parameters. - Fig. 2 also shows how sensitivity and specificity change with different cutoffs for these three parameters.
- Table 3 Prediction performance characteristics for impedance planimetry measurements most strongly associated with a clinical response (Eckardt score $\leq 3$ ).

\begin{tabular}{|c|c|c|c|c|}
\hline $\begin{array}{l}\text { Characteristic } \\
\text { and cutoff }\end{array}$ & $\begin{array}{l}\text { Sensitivi- } \\
\text { ty, \% }\end{array}$ & $\begin{array}{l}\text { Specifici- } \\
\text { ty, \% }\end{array}$ & $\begin{array}{l}\text { PPV, } \\
\%\end{array}$ & $\begin{array}{l}\text { NPV, } \\
\%\end{array}$ \\
\hline \multicolumn{5}{|l|}{$\Delta \mathrm{CSA}(\mathrm{mm})$} \\
\hline . 25 & 89.85 & 41.67 & 94.47 & 27.03 \\
\hline - 40 & 78.20 & 58.33 & 95.41 & 19.44 \\
\hline - 55 & 59.02 & 70.83 & 95.73 & 13.49 \\
\hline . 70 & 39.10 & 87.50 & 97.20 & 11.48 \\
\hline . 85 & 19.17 & 91.67 & 96.23 & 9.28 \\
\hline \multicolumn{5}{|l|}{$\% \triangle \mathrm{CSA}$} \\
\hline - 150 & 92.48 & 37.5 & 94.25 & 31.03 \\
\hline . 225 & 75.56 & 58.33 & 95.26 & 17.72 \\
\hline - 300 & 57.89 & 70.83 & 95.65 & 13.18 \\
\hline . 375 & 38.35 & 95.83 & 99.03 & 12.3 \\
\hline . 450 & 23.68 & 100.0 & 100.0 & 10.57 \\
\hline \multicolumn{5}{|l|}{$\% \Delta \mathrm{DI}$} \\
\hline . 150 & 88.72 & 37.5 & 94.02 & 23.08 \\
\hline . 225 & 74.44 & 54.17 & 94.74 & 16.05 \\
\hline - 300 & 63.16 & 79.17 & 97.11 & 16.24 \\
\hline . 375 & 47.37 & 87.5 & 97.67 & 13.04 \\
\hline . 450 & 38.72 & 87.5 & 97.17 & 11.41 \\
\hline
\end{tabular}

PPV, positive predictive value; NPV, negative predictive value; $\Delta$, change in parameter; CSA, cross-sectional area; $\% \Delta$, percent change in parameter; DI, distensibility index.

Performance characteristics at each cutoff are based on the complete data.

The optimal cutoff values based on the Yoden index for the four impedance planimetry measurements most predictive of a clinical response were as follows: $\triangle \mathrm{CSA}=46 \mathrm{~mm}$ (sensitivity $74 \%$, specificity $65 \%$ ); $\triangle \triangle C S A=360 \%$ (sensitivity $44 \%$, specificity $92 \%$ ); $\Delta \mathrm{DI}=1.84 \mathrm{~mm} / \mathrm{mmHg}$ (sensitivity $76 \%$, specificity $56 \%$ ); and $\% \Delta \mathrm{DI}=272 \%$ (sensitivity $69 \%$, specificity $72 \%$ ).

An exploratory multivariable GEE model for clinical response that included $\Delta P r, \Delta C S A, \% \Delta C S A$, and $\Delta D$ I as covariates was developed using GEEBoost for model selection followed by 10 -fold cross-validation to evaluate predictive performance $[13,14]$. The cvAUC for this model was 0.76 (95\%Cl $0.67-0.85)$, providing limited improvement in CvAUC compared with univariate models including $\triangle P r, \Delta C S A, \% \triangle C S A$, or $\Delta \mathrm{DI}$. Fig. $1 \mathrm{~s}$ shows the ROC curve and changes in sensitivity and specificity with increasing cutoffs for the predicted probability of a patient with a clinical response. 

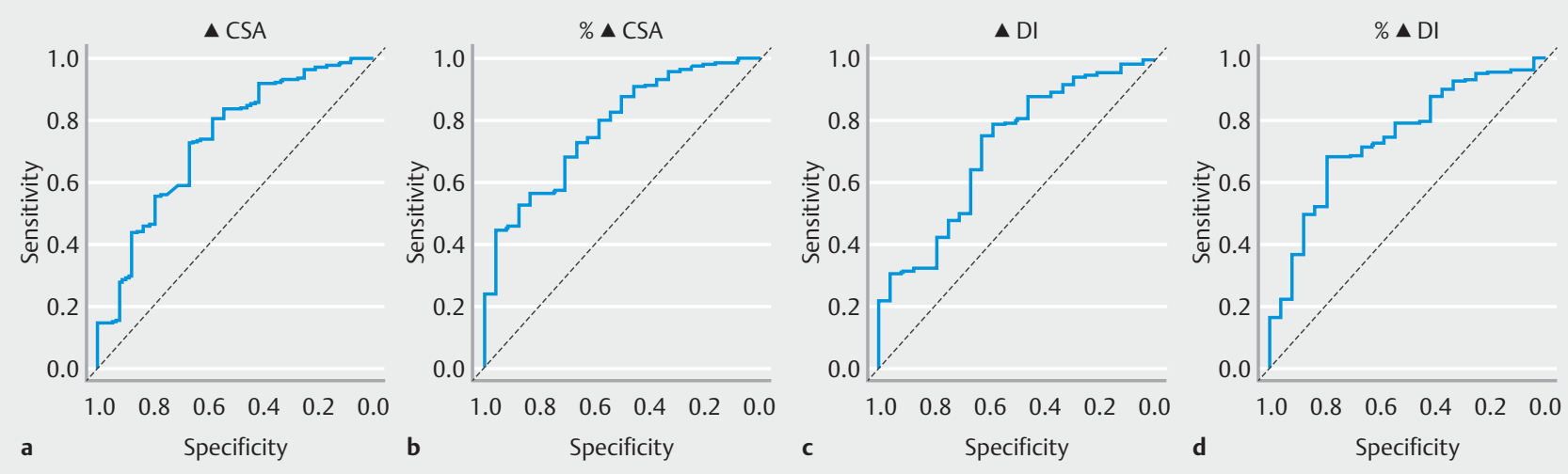

Fig. 1 Receiver operating characteristic (ROC) curves for the impedance planimetry parameters most predictive of a clinical response (Eckardt score $\leq 3)$. a Change in cross-sectional area $(\Delta C S A)$. b Percent change in cross-sectional area (\% $\Delta C S A)$. $\mathbf{c}$ Change in distensibility index $(\Delta \mathrm{DI})$. d Percent change in distensibility index $(\% \triangle \mathrm{DI})$. Black lines are the ROC curve for the generalized estimating equation model from the complete data and the grey lines are for the average ROC curve based on the 10 -fold cross-validated predictions.
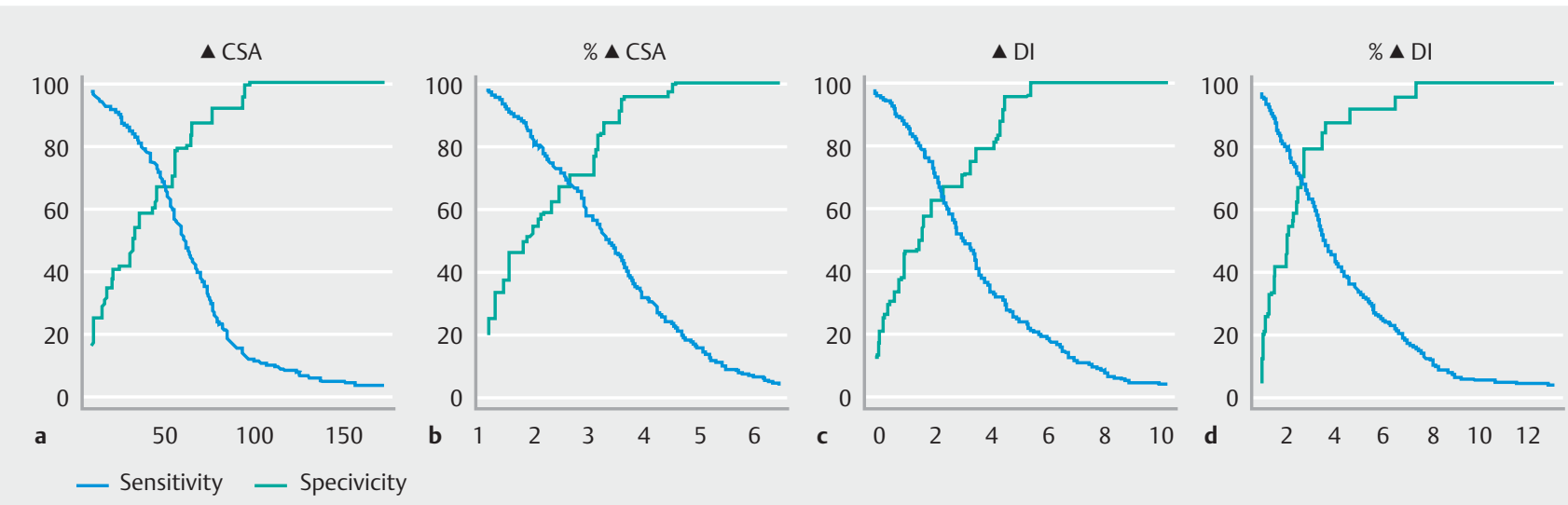

- Fig. 2 Sensitivity (solid line) and specificity (dashed line) with changing cutoffs for the impedance planimetry parameters most predictive of a clinical response (Eckardt score $\leq 3)$. a Change in cross-sectional area $(\triangle C S A)$. b Percent change in cross-sectional area (\% $\Delta C S A)$. $\mathbf{c} C h a n g e$ in distensibility index $(\Delta \mathrm{DI})$. $\mathbf{d}$ Percent change in distensibility index $(\% \Delta \mathrm{DI})$. The estimated sensitivity and specificity are based on the complete data.

\section{Prediction of abnormal pH impedance and/or esophagitis}

Repeat endoscopy or $\mathrm{pH}$ impedance testing was performed following POEM in 104 patients (35.9\%), 60 (57.7\%) of whom had abnormal $\mathrm{pH}$ impedance testing and/or esophagitis. The cvAUCs for $\mathrm{pH}$ impedance and/or esophagitis ranged from 0.40 to 0.62 , with the greatest observed cvAUCs for $\triangle C S A$ and $\% \triangle C S A$, indicating weak to moderate prediction performance ( $\triangleright$ Table 2 ). The ROC curves for $\triangle C S A$ and $\% \triangle C S A$ are presented in $>$ Fig.2s. $>$ Table 4 provides sensitivity, specificity, PPV, and NPV for different cutoffs for these two parameters, and $\nabla$ Fig. 2s also shows how sensitivity and specificity change with changing cutoffs for these two parameters.

\section{Prediction of post-POEM GERD symptoms}

Following POEM, data on the presence or absence of GERD symptoms was available for 274 patients, 108 (39.4\%) of whom reported the presence of GERD. The cvAUCs for occurrence of post-POEM GERD ranged from 0.43 to 0.62 , with the largest observed cVAUCs for post-POEM CSA and $\triangle$ CSA, indicating weak to moderate prediction performance ( $>$ Table 2 ). The ROC curves for post-POEM CSA and $\triangle$ CSA are presented in Fig. 3s. Table 4 provides sensitivity, specificity, PPV, and NPV for different cutoffs for these two parameters, and Fig.3s also shows how sensitivity and specificity change with changing cutoffs for these two parameters. 
- Table 4 Prediction performance characteristics for impedance planimetry measurements associated with abnormal pH/impedance and/or esophagitis, and with gastroesophageal reflux disease.

\begin{tabular}{|c|c|c|c|c|c|}
\hline Characteristic & Cutoff & Sensitivity, \% & Specificity, \% & PPV, \% & NPV, \% \\
\hline \multicolumn{6}{|c|}{ Abnormal pH impedance and/or esophagitis } \\
\hline \multirow[t]{5}{*}{ - $\triangle \mathrm{CSA}(\mathrm{mm})$} & 25 & 91.67 & 13.91 & 21.74 & 86.49 \\
\hline & 40 & 88.33 & 28.26 & 24.31 & 90.28 \\
\hline & 55 & 73.33 & 47.83 & 26.83 & 87.3 \\
\hline & 70 & 46.67 & 65.65 & 26.17 & 82.51 \\
\hline & 85 & 21.67 & 82.61 & 24.53 & 80.17 \\
\hline \multirow[t]{5}{*}{ - \% $\triangle \mathrm{CSA}$} & 150 & 95.00 & 11.3 & 21.84 & 89.66 \\
\hline & 225 & 83.33 & 30.00 & 23.7 & 87.34 \\
\hline & 300 & 70.00 & 48.26 & 26.09 & 86.05 \\
\hline & 375 & 48.33 & 67.83 & 28.16 & 83.42 \\
\hline & 450 & 26.67 & 79.57 & 25.4 & 80.62 \\
\hline \multicolumn{6}{|l|}{ GERD } \\
\hline \multirow[t]{5}{*}{ - Post-POEM CSA } & 60 & 95.37 & 17.03 & 40.55 & 86.11 \\
\hline & 80 & 75.93 & 43.41 & 44.32 & 75.24 \\
\hline & 100 & 41.67 & 72.53 & 47.37 & 67.69 \\
\hline & 120 & 21.3 & 84.07 & 44.23 & 64.29 \\
\hline & 140 & 11.11 & 90.11 & 40 & 63.08 \\
\hline \multirow[t]{5}{*}{ - $\Delta \mathrm{CSA}(\mathrm{mm})$} & 1.5 & 90.74 & 10.44 & 37.55 & 65.52 \\
\hline & 2.25 & 76.85 & 29.67 & 39.34 & 68.35 \\
\hline & 3 & 56.48 & 45.05 & 37.89 & 63.57 \\
\hline & 3.75 & 36.11 & 64.84 & 37.86 & 63.1 \\
\hline & 4.5 & 25 & 80.22 & 42.86 & 64.32 \\
\hline \multicolumn{6}{|c|}{$\begin{array}{l}\text { PPV, positive predictive value; NPV, negative predictive value; } \Delta, \text { change in parameter; CSA, cross-sectional area; } \% \Delta \text {, percent change in parameter; GERD, gastro } \\
\text { esophageal reflux disease; POEM, peroral endoscopic myotomy. } \\
\text { Performance characteristics at each cut-off are based on the complete data. }\end{array}$} \\
\hline
\end{tabular}

\section{Discussion}

In this international cohort analysis of 290 patients, the impedance planimetry measurements that were most predictive of a clinical response following POEM for treatment of achalasia were percent change in CSA (\% $\triangle \mathrm{CSA}$ ) and percent change in distensibility index $(\% \Delta \mathrm{DI})$.

Intraprocedural measurements that can predict the clinical response of endoscopic therapies for achalasia have the potential to enhance clinical outcomes by enabling real-time procedural modifications. Intraprocedural modifications during POEM could include lengthening the myotomy, conversion of a partial-thickness to a full-thickness myotomy or adding an additional myotomy in a different vector (anterior or posterior).

Rates of reflux esophagitis are firmly established as being higher in POEM compared with both laparoscopic Heller myotomy and pneumatic dilation, and this complication has the potential to scupper the long-term adoption of this procedure in favor of one of its alternatives $[15,16]$. If impedance planimetry can predict patients at risk of developing reflux due to excessive EG] distensibility, it will markedly impact clinical care, potentially allowing patients at high risk of GERD to undergo endoscopic treatments for GERD during the same session as POEM [17].

Increases in post-procedural CSA and distensibility index have been associated with a clinical response in studies evaluating the utility of post-procedural (laparoscopic Heller myotomy or POEM) impedance planimetry $[3,4,6]$. Based on these results, Wu et al. evaluated the utility of performing intraprocedural impedance planimetry in patients undergoing pneumatic dilation, to determine whether they would benefit from an upsizing of the pneumatic dilation during the same procedure [7]. A change of $>1.8 \mathrm{~mm}^{2} / \mathrm{mmHg}$ in the distensibility index following a $30-\mathrm{mm}$ pneumatic dilation was identified as the optimal cutoff for clinical response.

The parameters with the greatest ability to predict a clinical response, defined as those with the highest ROC, were $\% \Delta \mathrm{DI}$ 
and \% $\triangle \mathrm{CSA}$ with AUCs of $0.73(95 \% \mathrm{Cl} 0.63-0.82)$ and 0.75 ( $95 \% \mathrm{Cl} 0.67-0.89)$, respectively. The optimal cutoff between sensitivity and specificity was found to be a $\% \Delta \mathrm{DI}$ of $272 \%$, offering a sensitivity and specificity of $69 \%$ and $72 \%$, respectively, and a $\% \triangle$ CSA of $360 \%$ offering a sensitivity and specificity of $44 \%$ and $92 \%$, respectively. The finding that percent change was the most predictive impedance planimetry measurement for clinical response indicates that a relative increase is more predictive that an absolute increase or a single post-procedural value. Patients with very low preprocedural distensibility index may not need the same absolute change in impedance planimetry values as those with higher preprocedural index values to achieve a similar rate of clinical response.

Prior studies have shown that a higher post-procedural CSA and distensibility index are associated with GERD and reflux esophagitis. It is not possible to offer a meaningful reference range for CSA measurements in this study, as the different CSA values only had a low PPV for predicting both GERD and reflux esophagitis across a large spectrum of CSA values. This, combined with their low AUC, reflects the weak performance characteristics of these measurements for the above outcomes. More objective measures of reflux may yield different results; however, the weak predictive value of CSA is concerning for the overall ability of impedance planimetry to predict post-procedure reflux.

The published data evaluating how impedance planimetry may guide reflux therapies has been disappointing. Preoperative impedance planimetry measurements have not been predictive of a clinical response for surgical and endoscopic antireflux procedures $[18,19]$. The lack of an association has been attributed to differences in surgical techniques and the difficulty in evaluating functional disorders, namely, improvements in objective physiological measurements do not always correlate with improvements in subjective patient-centered clinical outcomes $[18,19]$.

An important strength of this study is the large multicenter cohort of patients, with multiple endoscopists performing POEM according to their differing center-specific protocols, allowing for more generalizable results. Given the overall high clinical success associated with achalasia treatments, a small sample size results in a very limited number of treatment failures on which to develop statistical models. Statistical inferences from these studies and "optimal cutoff values" must therefore be interpreted with caution owing to their low power.

A number of limitations exist for this study. Impedance planimetry measurements were only obtained directly after POEM. Measurements obtained at later time intervals, when EGJ remodeling and dilation occur may show improved performance characteristics. This approach would incur significant additional cost in the management of achalasia because of the need for repeat endoscopy. The present study lacked a validation cohort. Despite having a large cohort there were only 24 clinical failures. Splitting the present cohort into a test and a validation cohort would have substantially reduced the statistical power. Finally, clinical success was defined according to the Eckardt score, a clinical tool with only modest performance characteris- tics that has never been validated as an instrument for assessing clinical outcomes after achalasia treatment [20].

In conclusion, the percent change in both CSA and distensibility index were the strongest predictors of clinical success following POEM. Further studies are needed to determine whether intraprocedural modifications based on these results can improve clinical outcomes for patients undergoing POEM. The ability of impedance planimetry to predict reflux following POEM was weak.

\section{Competing interests}

B. Joseph Elmunzer is a consultant for Takeda Pharmaceuticals. Mouen A. Khashab is a consultant for Boston Scientific, Olympus, and Medtronic, and is also on the medical advisory board for Boston Scientific and Olympus. Vivek Kumbhari is a consultant for Apollo Endosurgery, Boston Scientific, Medtronic, and ReShape Life Science. Bu Hayee is a consultant for Apollo Endosurgery, Boston Scientific, Fuji, Medtronic, Obalon, Pentax Medical, and ReShape Life Sciences. All other authors declare that they have no conflicts of interest.

\section{References}

[1] Kim GH. Is EndoFLIP useful for predicting clinical outcomes after peroral endoscopic myotomy in patients with achalasia? Gut Liver 2019; 13: 3-4

[2] Rohof WO, Hirsch DP, Kessing BF et al. Efficacy of treatment for patients with achalasia depends on the distensibility of the esophagogastric junction. Gastroenterology 2012; 143: 328-335

[3] Ngamruengphong S, von Rahden BH, Filser J et al. Intraoperative measurement of esophagogastric junction cross-sectional area by impedance planimetry correlates with clinical outcomes of peroral endoscopic myotomy for achalasia: a multicenter study. Surg Endosc 2016; 30: 2886-2894

[4] Teitelbaum EN, Boris L, Arafat FO et al. Comparison of esophagogastric junction distensibility changes during POEM and Heller myotomy using intraoperative FLIP. Surg Endosc 2013; 27: 4547-4555

[5] Teitelbaum EN, Soper NJ, Pandolfino JE et al. Esophagogastric junction distensibility measurements during Heller myotomy and POEM for achalasia predict postoperative symptomatic outcomes. Surg Endosc 2015; 29: 522-528

[6] Yoo IK, Choi SA, Kim WH et al. Assessment of clinical outcomes after peroral endoscopic myotomy via esophageal distensibility measurements with the endoluminal functional lumen imaging probe. Gut Liver 2019; 13: 32-39

[7] Wu PI, Szczesniak MM, Craig Pl et al. Novel intra-procedural distensibility measurement accurately predicts immediate outcome of pneumatic dilatation for idiopathic achalasia. Am J Gastroenterol 2018; 113: 205-212

[8] Kahrilas PJ, Bredenoord A], Fox M et al. The Chicago Classification of esophageal motility disorders, v3.0. . Neurogastroenterol Motil 2015; 27: $160-174$

[9] Khashab MA, El Zein M, Kumbhari V et al. Comprehensive analysis of efficacy and safety of peroral endoscopic myotomy performed by a gastroenterologist in the endoscopy unit: a single-center experience. Gastrointest Endosc 2016; 83: 117-125

[10] Eckardt VF, Aignherr C, Bernhard G. Predictors of outcome in patients with achalasia treated by pneumatic dilation. Gastroenterology 1992; 103: $1732-1738$ 
[11] Lundell LR, Dent J, Bennett JR et al. Endoscopic assessment of oesophagitis: clinical and functional correlates and further validation of the Los Angeles classification. Gut 1999; 45: 172-180

[12] LeDell E, Petersen M, van der Laan M. Computationally efficient confidence intervals for cross-validated area under the ROC curve estimates. Electron J Stat 2015; 9: 1583-1607

[13] Wolfson J. EEBoost: a general method for prediction and variable selection based on estimating equations. J Am Stat Assoc 2011; 106: 296-305

[14] Brown B, Miller C], Wolfson J. ThrEEBoost: thresholded boosting for variable selection and prediction via estimating equations. J Comput Graph Stat 2017; 26: 579-588

[15] Repici A, Fuccio L, Maselli R et al. GERD after per-oral endoscopic myotomy as compared with Heller's myotomy with fundoplication: a systematic review with meta-analysis. Gastrointest Endosc 2018; 87: 934-943.e918
[16] Werner YB, Hakanson B, Martinek J et al. Endoscopic or surgical myotomy in patients with idiopathic achalasia. N Engl J Med 2019; 381: 2219-2229

[17] Pandolfino JE, Shi G, Curry J et al. Esophagogastric junction distensibility: a factor contributing to sphincter incompetence. Am J Physiol Gastrointest Liver Physiol 2002; 282: G1052-1058

[18] Smeets FG, Keszthelyi D, Masclee AA et al. Measurement of esophagogastric junction distensibility may assist in selecting patients for endoluminal gastroesophageal reflux disease surgery: author's reply. J Neurogastroenterol Motil 2015; 21: 450-451

[19] Perretta S, McAnena O, Botha A et al. Acta from the EndoFLIPâ Symposium. Surg Innov 2013; 20: 545-552

[20] Taft TH, Carlson DA, Triggs ] et al. Evaluating the reliability and construct validity of the Eckardt symptom score as a measure of achalasia severity. Neurogastroenterol Motil 2018; 30: e13287 